\title{
Stability of fluid flow past a membrane
}

\author{
V. Kumaran ${ }^{\mathrm{a}}$ and L. Srivatsan \\ Department of Chemical Engineering, Indian Institute of Science, Bangalore 560 012, India
}

\begin{abstract}
The stability of the flow of a fluid past a solid membrane of infinitesimal thickness is investigated using a linear stability analysis. The system consists of two fluids of thicknesses $R$ and $H R$ and bounded by rigid walls moving with velocities $V_{\mathrm{a}}$ and $V_{\mathrm{b}}$, and separated by a membrane of infinitesimal thickness which is flat in the unperturbed state. The fluids are described by the Navier-Stokes equations, while the constitutive equation for the membrane incorporates the surface tension, and the effect of curvature elasticity is also examined for a membrane with no surface tension. The stability of the system depends on the dimensionless strain rates $\Lambda_{\mathrm{a}}$ and $\Lambda_{\mathrm{b}}$ in the two fluids, which are defined as $\left(V_{\mathrm{a}} \eta / \Gamma\right)$ and $\left(-V_{\mathrm{b}} \eta / \Gamma H\right)$ for a membrane with surface tension $\Gamma$, and $\left(V_{\mathrm{a}} R^{2} \eta / K\right)$ and $\left(V_{\mathrm{b}} R^{2} \eta / K H\right)$ for a membrane with zero surface tension and curvature elasticity $K$. In the absence of fluid inertia, the perturbations are always stable. In the limit $k \rightarrow 0$, the decay rate of the perturbations is $O\left(k^{3}\right)$ smaller than the frequency of the fluctuations. The effect of fluid inertia in this limit is incorporated using a small wave number $k \ll 1$ asymptotic analysis, and it is found that there is a correction of $O(k \mathrm{Re})$ smaller than the leading order frequency due to inertial effects. This correction causes long wave fluctuations to be unstable for certain values of the ratio of strain rates $\Lambda_{\mathrm{r}}=\left(\Lambda_{\mathrm{b}} / \Lambda_{\mathrm{a}}\right)$ and ratio of thicknesses $H$. The stability of the system at finite Reynolds number was calculated using numerical techniques for the case where the strain rate in one of the fluids is zero. The stability depends on the Reynolds number for the fluid with the non-zero strain rate, and the parameter $\Sigma=\left(\rho \Gamma R / \eta^{2}\right)$, where $\Gamma$ is the surface tension of the membrane. It is found that the Reynolds number for the transition from stable to unstable modes, $\mathrm{Re}_{\mathrm{t}}$, first increases with $\Sigma$, undergoes a turning point and a further increase in the $\mathrm{Re}_{\mathrm{t}}$ results in a decrease in $\Sigma$. This indicates that there are unstable perturbations only in a finite domain in the $\Sigma-\mathrm{Re}_{\mathrm{t}}$ plane, and perturbations are always stable outside this domain.
\end{abstract}

PACS. 47.15.Fe Stability of laminar flows $-47.60 .+\mathrm{i}$ Flows in ducts, channels, nozzles, and conduits

\section{Introduction}

Fluid flow adjacent to a flexible surface is often encountered in biological systems and biotechnology processes. The flow of blood and other fluids in the body takes place through tubes whose walls are made of elastic materials such as tissues and membranes. Such flows are also encountered in industrial applications such as hollow fibre reactors and membrane bioreactors. Separation and purification processes in pharmaceutical industries often involve flow and diffusion of a fluid in tubes and channels made up of polymer matrices and membranes. In these systems, it is of interest to analyse the effect of the wall flexibility on the flow structure, in order to accurately describe the transport processes. In the present analysis, the flow of a fluid adjacent to a solid membrane of infinitesimal thickness is considered, and the effect of the flexibility of the membrane on the stability of the flow is analysed.

There has been some work, both experimental [1] as well as theoretical [2-4] on the surface hydrodynamics of

\footnotetext{
a e-mail: kumaran@chemeng.iisc.ernet.in
}

polymer gels and membranes. In most of the previous theoretical studies on the surface modes of complex fluids, the dispersion relation is calculated using a single fluid model for the bulk properties. In this approach, the polymer is treated as a viscoelastic fluid described by the nonNewtonian Navier-Stokes equations, in which the shear stress is a non-linear function of the shear rate. A two fluid model was used by Harden, Pleiner and Pincus [2], who wrote coupled equations for the fluid velocity and polymer displacement field in a polymer gel. The equations were solved in the infinite coupling limit, where the coupling constant between the fluid and the network is large, so the velocities are assumed to be equal in the leading approximation. In addition to the viscous stress due to the fluid flow, there is also an elastic stress due to straining of the network. This gave rise to novel features that were not observed in previous studies. In particular, there is a time scale associated with elastic relaxation of the polymer matrix which causes unusual correlations in the surface fluctuation. The surface modes on a gel of finite thickness was analysed by Kumaran [3], in the limit where the elastic oscillation time for the strain field 
is small compared to the viscous relaxation time. In this limit, the dominant forces in the momentum conservation equation are the elastic and inertial forces, and the viscous forces cause a subdominant correction. There are multiple frequencies of oscillation, and the decay rate of the fluctuations is small compared to the frequency of oscillation. In addition, it was found that the surface fluctuations are sensitive not just to the conditions at the free surface, but also to the conditions at the other (fixed) surface. There are significant differences between "grafted" gels, where the gel is fixed onto a rigid surface, and "adsorbed" gels, where the network can move laterally at the fixed surface. The hydrodynamic modes on a viscoelastic film of polymeric material at the interface between two Newtonian fluids was analysed by Harden and Pleiner [4]. The dispersion relations and structure factor for the surface oscillations were obtained using generalised boundary conditions derived for a thin viscoelastic surface. The coupling between the transverse modes and the in plane longitudinal modes leads to certain unusual features of the surface fluctuations.

There have also been studies on the effect of fluid flow on the fluctuations at the surface of polymer gels. Kumaran, Fredrickson and Pincus [5] analysed the stability of the interface between a gel and a fluid at low Reynolds number in the absence of inertia, and found that there could be an instability when the strain rate in the fluid is increased beyond a critical value. This analysis has been extended to finite Reynolds number using numerical techniques by Srivatsan and Kumaran [6]. There have also been studies on the stability of the flow of a fluid in a tube with gel walls [7], and these studies have concluded that the flexibility of the wall destabilises the flow. A similar conclusion was reached in the experimental studies of Krindel and Silberberg [8] on the flow through a gel-walled tube. In the present analysis, we consider the stability of the flow of a Newtonian fluid adjacent to a membrane of infinitesimal thickness. This is different from the instabilities between two fluids caused due to discontinuities in the strain rates in the two fluids. The boundary conditions at an interface between two fluids require that the velocity and the stress in the tangential and normal direction are continuous at the interface. In the present case, there is a solid membrane at the interface, and it is shown a little later that the tangential velocity at the membrane is zero in the leading order approximation when the normal displacement is small compared to the wavelength of the fluctuations. Consequently, there is no tangential stress continuity condition, while the normal velocity and stress are continuous across the membrane.

The analysis of the stability in the low Reynolds number limit in Section 2.1 indicates that the fluctuations are always stable in the absence of fluid inertia. It is found that in the limit $k \rightarrow 0$, the decay rate of the fluctuations is $O\left(k^{3}\right)$ smaller than the frequency. However, there is a correction to the growth rate which is $O(k)$ smaller than the leading order frequency due to inertial effects. This is determined using a low wave number analysis in Section 2.2, and it is found that small wave number fluctu-

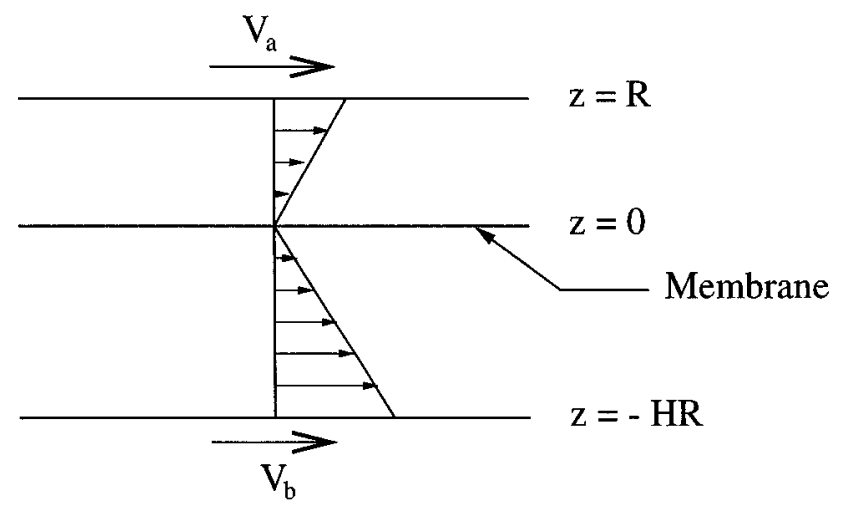

Fig. 1. Configuration and coordinate system of the fluidmembrane-fluid system.

ations are unstable for certain values of the ratio of strain rates $\left(\Lambda_{\mathrm{b}} / \Lambda_{\mathrm{a}}\right)$, and for certain values of the ratio of thicknesses $H$. The calculation is extended to intermediate values of the Reynolds number using numerical techniques in Section 2.3. In Section 2.4, a membrane with zero surface tension but non zero curvature elasticity is considered, and the asymptotic results are derived for this case. The main results are briefly discussed in Section 3.

\section{Analysis}

The system consists of a membrane of infinitesimal thickness, surface tension $\Gamma$, and negligible inertia stretched along the interface $z=0$ between two fluids $A$ and $B$ of thickness $R$ and $H R$, density $\rho$ and viscosity $\eta$ as shown in Figure 1. For simplicity, the densities and viscosities of the two fluids are equal; a variation in the densities and viscosities will cause a quantitative change in the stability characteristics, but the qualitative behaviour will be the same. The effect of curvature on the normal stress in the membrane is neglected because it turns out that the most interesting behaviour is encountered in limit of long wavelength, and curvature effects are not important in this limit. The surface bounding the fluid $A$ at $z=R$ moves with a velocity $V_{\mathrm{a}}$, while the surface bounding the fluid $B$ at $z=-H R$ moves with a velocity $V_{\mathrm{b}}$. The lengths in the problem are scaled by the thickness $R$, the velocities by $(\Gamma / \eta)$ and the time coordinate by $(R \eta / \Gamma)$. The equations for the fluid are the Navier-Stokes equations:

$$
\begin{aligned}
\partial_{i} v_{i}^{n} & =0 \\
\left(\operatorname{Re} / \Lambda_{\mathrm{a}}\right)\left(\partial_{\mathrm{t}} v_{i}^{n}+v_{j}^{n} \partial_{j} v_{i}^{n}\right) & =-\partial_{i} p^{n}+\partial_{j}^{2} v_{i}^{n}
\end{aligned}
$$

where $\partial_{\mathrm{t}} \equiv(\partial / \partial t), \partial_{i} \equiv\left(\partial / \partial x_{i}\right)$, the indices $i$ and $j$ represent the components of a vector, and repeated indices represent a dot product. The superscript $n$ in equations $(1,2)$ is a for the fluid $\mathrm{A}$ in the region $z>0$, and $\mathrm{b}$ for the fluid $\mathrm{B}$ in the region $z<0$. In equation (2), the Reynolds number is defined as $\operatorname{Re}=\left(\rho V_{\mathrm{a}} R / \eta\right)$, and the parameters $\Lambda_{\mathrm{a}}=\left(V_{\mathrm{a}} \eta / \Gamma\right)$ and $\Lambda_{\mathrm{b}}=\left(-V_{\mathrm{b}} \eta / \Gamma H\right)$. The scaled mean 


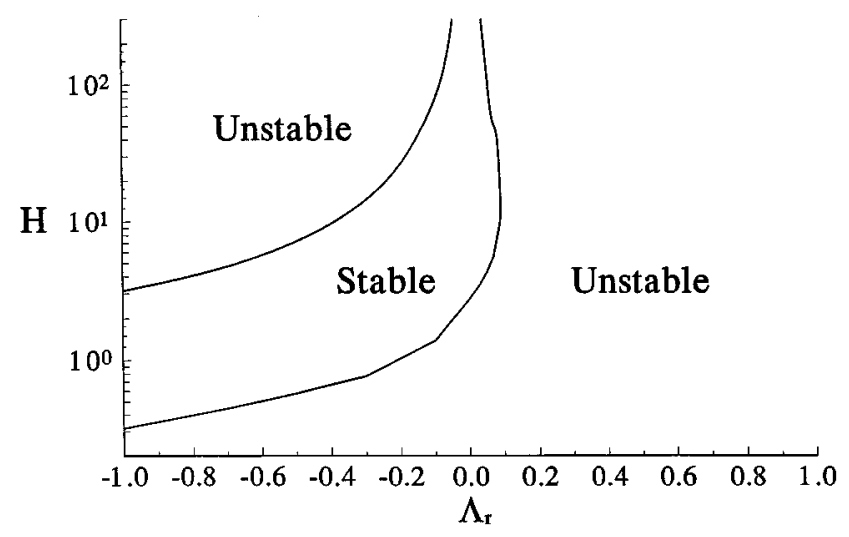

Fig. 2. Domains in the $\Lambda_{\mathrm{r}}-H$ plane where fluid inertia has stabilising and destabilising effects in the low wave number limit.

velocities for $z>0$ and $z<0$ are:

$$
\bar{v}_{x}^{n}=\Lambda_{n} z
$$

where $n$ is $a$ for fluid $A$, and $n$ is $b$ for fluid $B$. The boundary conditions at the surfaces $z=1$ and $z=-H$ are the no-slip conditions, which require that the normal velocity at these surfaces is zero, while the tangential velocity is equal to the surface velocity.

The constitutive equation relating the normal stress and normal displacement for the membrane incorporates the effect of surface tension, but the stress due to curvature was neglected because this effect is small in the limit of small wave number, which turns out to be the most interesting limit for this system. In addition, the tangential velocity in the fluid is set equal to zero at the membrane surface.

$$
v_{x}^{n}=0 \text { for } n=\mathrm{a}, \mathrm{b} .
$$

This is because the tangential displacement of the membrane surface is small compared to the normal displacement when the normal displacement is large compared to the membrane thickness. For example, if the membrane is infinitesimally thick and the amplitude and wavelength of the perturbations are $u$ and $\lambda$ respectively, then the extension along the surface of the membrane is proportional to $\left(u^{2} / \lambda\right)$, which is small compared to $u$ for $u \ll \lambda$. Consequently, the no-slip condition requires that the tangential velocity in the fluid is also $O(u / \lambda)$ smaller than the normal velocity, and is set equal to zero in the leading approximation.

The normal velocity boundary condition states that the normal velocity of the fluid at the membrane is equal to the velocity of the membrane itself, while the normal stress is balanced across the surface.

$$
v_{z}^{\mathrm{a}}=v_{z}^{\mathrm{b}}=\partial_{\mathrm{t}} u \quad \tau_{z z}^{\mathrm{a}}=\tau_{z z}^{\mathrm{b}}-\Gamma \partial_{x}^{2} u
$$

where $u$ is the normal displacement, and $\Gamma$ is the surface tension of the membrane.

In the linear stability analysis, small perturbations are imposed on the mean velocity fields in the fluids $A$ and

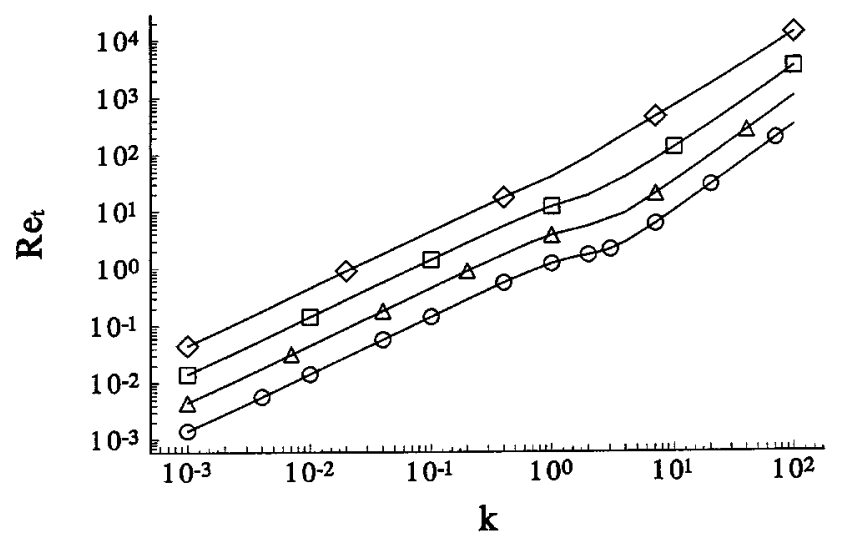

Fig. 3. Variation of the transition Reynolds number, $\mathrm{Re}_{\mathrm{t}}$, as a function of wave number, $k$, for $H=1$. (०) $\Sigma=0.1 ;(\triangle)$ $\Sigma=1.0 ;(\square) \Sigma=10.0 ;(\diamond) \Sigma=100.0$.

$B$ and the normal displacement of the membrane of the form:

$$
\begin{aligned}
v_{i}^{n}(x, z, t) & =\bar{v}_{i}^{n}(z)+\tilde{v}_{i}^{n}(z) \exp (i k x+\alpha t), \\
u & =\tilde{u} \exp (i k x+\alpha t) .
\end{aligned}
$$

These perturbed fields are inserted into the mass and momentum equations $(1,2)$, and only terms linear in the perturbations are retained, to obtain the following linearised equations for the velocity fields $\tilde{v}_{i}^{\mathrm{a}}$ and $\tilde{v}_{i}^{\mathrm{b}}$ :

$$
\mathrm{d}_{z} \tilde{v}_{z}^{n}+i k \tilde{v}_{x}^{n}=0
$$

$$
\begin{array}{r}
-i k \tilde{p}^{n}+\left[-\left(\operatorname{Re} / \Lambda_{\mathrm{a}}\right)\left(\alpha+\Lambda_{n} i k z\right)+\mathrm{d}_{z}^{2}-k^{2}\right] \tilde{v}_{x}^{n} \\
-\left(\operatorname{Re} \Lambda_{n} / \Lambda_{\mathrm{a}}\right) \tilde{v}_{z}^{n}=0
\end{array}
$$

$$
-\mathrm{d}_{z} \tilde{p}^{n}+\left[-\left(\operatorname{Re} / \Lambda_{\mathrm{a}}\right)\left(\alpha+\Lambda_{n} i k z\right)+\mathrm{d}_{z}^{2}-k^{2}\right] \tilde{v}_{z}^{n}=0
$$

where $\mathrm{d}_{z} \equiv(\mathrm{d} / \mathrm{d} z)$. A fourth order differential equation for $\tilde{v}_{z}^{n}$ is obtained by adding $\left(-\mathrm{d}_{z} \times\right.$ (Eq. (8)) $+i k \times$ (Eq. (9)), and using equation (7) to express $\tilde{v}_{x}^{n}$ in terms of $\tilde{v}_{z}^{n}$ :

$$
\left[-\left(\alpha+\Lambda_{n} i k z\right)\left(\operatorname{Re} / \Lambda_{\mathrm{a}}\right)+\mathrm{d}_{z}^{2}-k^{2}\right]\left(\mathrm{d}_{z}^{2}-k^{2}\right) \tilde{v}_{z}^{n}=0 .
$$

The perturbations to the stress fields in the fluid are:

$$
\tilde{\tau}_{x z}^{n}=\mathrm{d}_{z} \tilde{v}_{x}^{n}+i k \tilde{v}_{z}^{n} \quad \tilde{\tau}_{z z}^{n}=-\tilde{p}^{n}+2 \mathrm{~d}_{z} \tilde{v}_{z}^{n} .
$$

The boundary conditions are derived by inserting the equations for the velocity and displacement (6) into the boundary conditions $(4,5)$. The boundary conditions at the surfaces $z=1$ and $z=-H$ are:

$$
\tilde{v}_{z}^{n}=0, \quad \tilde{v}_{x}^{n}=0 .
$$

It is necessary to apply the matching conditions $(4,5)$ at the perturbed interface of the membrane $z=u$. However, the velocity and stress fields at the perturbed surface can 
be expressed in terms of the velocity and stress at the unperturbed surface $z=0$ using a Taylor series expansion, and only the linear terms in the expansion retained, to provide the following boundary conditions at the unperturbed surface $z=0$ :

$$
\begin{gathered}
\tilde{v}_{x}^{n}+\Lambda_{n} \tilde{u}=0 \\
\tilde{v}_{z}^{\mathrm{a}}=\tilde{v}_{z}^{\mathrm{b}}=\alpha \tilde{u} \tilde{\tau}_{z z}^{\mathrm{a}}=\tilde{\tau}_{z z}^{\mathrm{b}}+k^{2} \tilde{u} .
\end{gathered}
$$

In equation (13), the second term on the left side represents the variation in the mean velocity at the surface due to the displacement of the surface.

The growth rate $\alpha$ is obtained by solving the differential equations $(7,8,9)$ subject to the boundary conditions $(13,14)$. In this section, the stability of the system in the absence of fluid inertia is studied using low Reynolds number asymptotic analysis. It is found that the system is always stable in the absence of inertia. The effect of inertia in the low Reynolds number limit is studied using a low wave number analysis. The low Reynolds number asymptotic calculations are then extended to the finite Reynolds number regime using numerical methods.

\subsection{Low Reynolds number analysis}

In this analysis, we set $\operatorname{Re}=0$ in the governing equations for $\tilde{v}_{z}^{\mathrm{a}}$ and $\tilde{v}_{z}^{\mathrm{b}}$. The solutions of the resulting equations for $\tilde{v}_{z}^{\mathrm{a}}$ and $\tilde{v}_{z}^{\mathrm{b}}$ that are consistent with the boundary conditions at the top and bottom plates are:

$$
\begin{aligned}
& \tilde{v}_{z}^{\mathrm{a}}= a_{1}\left[\mathrm{e}^{-k z}-(1+2 k-2 k z) \mathrm{e}^{k z-2 k}\right] \\
&+a_{2}\left[z \mathrm{e}^{-k z}+(2 k z-2 k-z) \mathrm{e}^{k z-2 k}\right], \\
& \\
& \tilde{v}_{z}^{\mathrm{b}}=b_{1}\left[z \mathrm{e}^{k z}+(2 k-1) \mathrm{e}^{k z} /(2 k)+\mathrm{e}^{-(k z+2 k H)} /(2 k)\right] \\
&+b_{2}\left[z \mathrm{e}^{-k z}+(2 k H+1) \mathrm{e}^{-k z} /(2 k)-\mathrm{e}^{k z+2 k H} /(2 k)\right] .
\end{aligned}
$$

The tangential velocities $\tilde{v}_{x}^{\mathrm{a}}$ and $\tilde{v}_{x}^{\mathrm{b}}$ are obtained using the mass conservation equation, and the pressures $\tilde{p}^{\mathrm{a}}$ and $\tilde{p}^{\mathrm{b}}$ are obtained from the momentum conservation equation in the $x$-direction. These expressions are inserted in the matching conditions at the interface, i.e in (13) and (14), and a matrix equation of the form

$$
\mathbf{A} \mathbf{C}=\mathbf{0} \text {, }
$$

is obtained. Here, $\mathbf{C}=\left[a_{1}, a_{2}, b_{1}, b_{2}\right]$ is an array of constants. The determinant of this matrix is set equal to zero to obtain the characteristic equation. The characteristic equation turns out to be linear in the growth rate $\alpha$, and this can easily be solved to obtain an analytical expression for $\alpha$. This analytical expression is rather complex, and so it is not provided in its full form. The expression indicates that the perturbations are always stable at all wave numbers and for all ratio of thicknesses $H$. In the limit $k \rightarrow 0$, the first three terms in the Taylor series expansion for the growth rate $\alpha$ are

$$
\begin{aligned}
\alpha= & \frac{-i H k\left(H^{2} \Lambda_{\mathrm{a}}-\Lambda_{\mathrm{b}}\right)}{2\left(H^{3}+1\right)} \\
& +\frac{i k^{3} H^{3}\left[2\left(\Lambda_{\mathrm{a}}+\Lambda_{\mathrm{b}} H\right)(1-H)+5\left(\Lambda_{\mathrm{a}} H^{2}-\Lambda_{\mathrm{b}}\right)\right]}{30\left(H^{3}+1\right)\left(H^{2}-H+1\right)} \\
& -\frac{H^{3} k^{4}}{12\left(H^{3}+1\right)}+O\left(k^{5}\right) .
\end{aligned}
$$

The above equation indicates that the decay rate of the fluctuations is $O\left(k^{3}\right)$ smaller than the frequency in the limit $k \rightarrow 0$. However, in the limit of low wave number, the correction to the velocity field due to inertial terms is $O(k)$, and so the inertial correction to the growth rate is larger than the $O\left(k^{4}\right)$ damping due to viscous terms in the limit $k \rightarrow 0$. Consequently, it is of interest to examine the effect of this inertial correction on the stability of the system. This is determined using a low wave number analysis in the next subsection.

\subsection{Low wave number analysis}

In the present section, a low wave number asymptotic analysis is used to examine the effect of fluid inertia on the stability in the limit $k \rightarrow 0$.

Before proceeding with the analysis, it is useful to examine the magnitudes of the velocity and pressure fields in the limit $k \rightarrow 0$. As mentioned above, the leading order growth rate is $O(k)$ in this limit. If $\tilde{v}_{x}$ scales as $\tilde{v}$, then it follows from the continuity equations that $\tilde{v}_{z}$ scales as $k \tilde{v}$, and from the momentum conservation equation in the $x$-direction it can be seen that $\tilde{p}$ scales as $(\tilde{v} / k)$. The growth rate and the velocity and pressure fields are expanded in an asymptotic series in $k$, and the first two terms in the expansion consistent with the scaling considerations outlined above are:

$$
\begin{gathered}
\alpha=k \alpha^{0}+k^{2} \alpha^{1}+\ldots, \\
\tilde{v}_{z}^{n}=k \tilde{v}_{z}^{n 0}+k^{2} \tilde{v}_{z}^{n 1}+\ldots, \\
\tilde{v}_{x}^{n}=\tilde{v}_{x}^{n 0}+k \tilde{v}_{x}^{n 1}+\ldots \\
\tilde{p}^{n}=k^{-1} \tilde{p}^{n 0}+\tilde{p}^{n 1}+\ldots
\end{gathered}
$$

The governing equation (10) is expanded in a Taylor series in the wave number $k$, and the leading order and $O(k)$ correction to the governing equations are

$$
\mathrm{d}_{z}^{4} \tilde{v}_{z}^{n 0}=0
$$

and for $\tilde{v}_{z}^{\mathrm{a} 1}$ and $\tilde{v}_{z}^{\mathrm{b} 1}$

$$
\mathrm{d}_{z}^{4} \tilde{v}_{z}^{n 1}=\left(\operatorname{Re} / \Lambda_{\mathrm{a}}\right)\left(\alpha^{0}+i \Lambda_{n} z\right) \mathrm{d}_{z}^{2} \tilde{v}_{z}^{n 0}
$$

The leading order pressure terms are obtained from the equations

$$
\tilde{p}^{n 0}=-i \mathrm{~d}_{z}^{2} \tilde{v}_{x}^{n 0}
$$




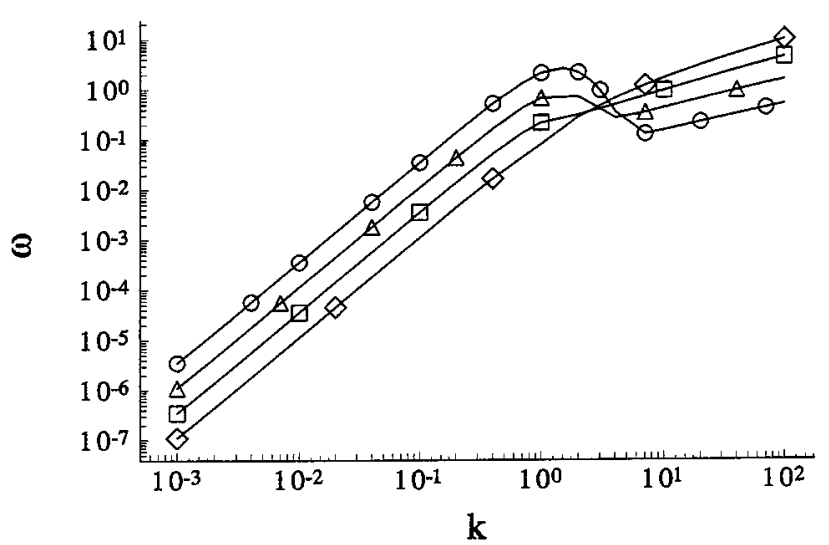

Fig. 4. Variation of the frequency, $\omega$, as a function of wave number, $k$, for $H=1$. (०) $\Sigma=0.1$; $\triangle$ ) $\Sigma=1.0$; ( $\square) \Sigma=10.0$; $(\diamond) \Sigma=100.0$.

and the corrections to the pressure terms are obtained from

$$
\begin{aligned}
\tilde{p}^{n 1}= & -i\left[\mathrm{~d}_{z}^{2} \tilde{v}_{x}^{n 1}-\left(\operatorname{Re} / \Lambda_{\mathrm{a}}\right)\left(\alpha^{0}+i \Lambda_{\mathrm{a}} z\right) \tilde{v}_{x}^{\mathrm{a} 0}\right. \\
& \left.-\left(\operatorname{Re} \Lambda_{n} / \Lambda_{\mathrm{a}}\right) \tilde{v}_{z}^{\mathrm{a} 0}\right]
\end{aligned}
$$

The leading order normal velocities $\tilde{v}_{z}^{\mathrm{a} 0}$ and $\tilde{v}_{z}^{\mathrm{b} 0}$ are obtained by integrating equations (19). The solutions of these differential equations that are consistent with the boundary conditions at the top and bottom plates are:

$$
\begin{aligned}
& \tilde{v}_{z}^{\mathrm{a} 0}=a_{1}(z-1)^{2}+a_{2}(z-1)^{2}(z+2) \\
& \tilde{v}_{z}^{\mathrm{b} 0}=b_{1}(z+H)^{2}+b_{2}(z+H)^{2}(z-2 H) .
\end{aligned}
$$

The leading order tangential velocities $\tilde{v}_{x}^{\mathrm{a} 0}$ and $\tilde{v}_{x}^{\mathrm{b} 0}$ are obtained by substituting equations (23) into the continuity equations. They are

$$
\begin{aligned}
& \tilde{v}_{x}^{\mathrm{a} 0}=2 i a_{1}(z-1)+3 i a_{2}\left(z^{2}-1\right) \\
& \tilde{v}_{x}^{\mathrm{b} 0}=2 i b_{1}(z+H)+3 i b_{2}\left(z^{2}-H^{2}\right) .
\end{aligned}
$$

The first correction to the normal velocities $\tilde{v}_{z}^{\mathrm{a} 1}$ and $\tilde{v}_{z}^{\mathrm{b} 1}$ are obtained by integrating equations (20), while the first corrections to the tangential velocity and pressure are obtained from the mass conservation and $x$ momentum conservation equations. Substituting these expressions into the matching conditions at the interface, i.e in $(13,14)$, a matrix of the following form is obtained:

$$
\mathbf{A} \mathbf{C}=\mathbf{0} \text {, }
$$

where $\mathbf{C}=\left[a_{1}, a_{2}, b_{1}, b_{2}\right]$. This characteristic equation, which is obtained by setting the determinant of $\mathbf{A}$ equal to zero, has the form:

$$
f\left(\alpha^{0}\right) k+g\left(\alpha^{0}, \alpha^{1}\right) k^{2}+\ldots=0
$$

where $f$ is the leading order term of the determinant and $g$ is its first correction. The leading order, first order and

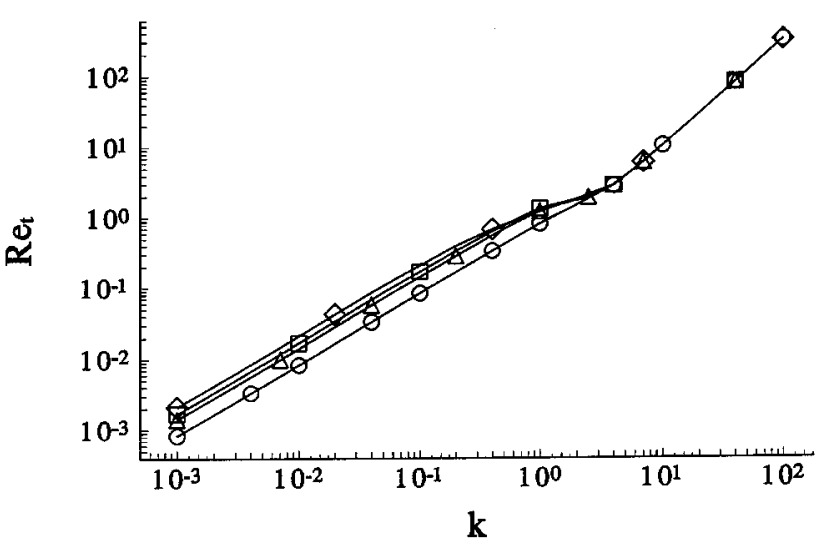

Fig. 5. Variation of the transition Reynolds number, $\mathrm{Re}_{\mathrm{t}}$, as a function of wave number, $k$, for $\Sigma=0.1$. (०) $H=0.5 ;(\triangle)$ $H=1.0 ;(\square) H=1.5 ;(\diamond) H=2.0$.

higher order correction terms of the determinant should separately be zero for the determinant to be zero, i.e

$$
f\left(\alpha^{0}\right)=0, \quad g\left(\alpha^{0}, \alpha^{1}\right)=0, \quad \ldots
$$

Solving these simultaneous equations, we get

$$
\alpha^{0}=-\frac{i H\left(H^{2} \Lambda_{\mathrm{a}}-\Lambda_{\mathrm{b}}\right)}{2\left(H^{3}+1\right)},
$$

$$
\begin{aligned}
\alpha^{1}= & -\frac{\Lambda_{\mathrm{a}} \operatorname{Re} H^{3}}{120\left(H^{3}+1\right)^{2}\left(H^{2}-H+1\right)} \\
& \times\left[\left(H^{5}-4 H^{4}+4 H^{3}-2 H^{2}+2 H-2\right)\right. \\
& -6 H \Lambda_{\mathrm{r}}\left(H^{4}-H^{3}+H^{2}-H+1\right) \\
& \left.+H \Lambda_{\mathrm{r}}^{2}\left(1-4 H+4 H^{2}-2 H^{3}+2 H^{4}-2 H^{5}\right)\right]
\end{aligned}
$$

where $\Lambda_{\mathrm{r}}=\Lambda_{\mathrm{b}} / \Lambda_{\mathrm{a}}$. Equation (29) for $\alpha^{1}$ indicates that the $O\left(k^{2}\right)$ correction to the growth rate due to inertial effects is real, and could be positive or negative depending on the parameter values of $H$ and $\Lambda_{\mathrm{r}}$. The parameter regimes where the perturbations are stable or unstable is shown in the $\Lambda_{\mathrm{r}}-H$ plane in Figure 2. Only the parameter range for $-1 \leq \Lambda_{\mathrm{r}} \leq 1$ has been shown because the results for $\Lambda_{\mathrm{r}}<-1$ and $\Lambda_{\mathrm{r}}>1$ can be deduced from symmetry. The results indicate that there are domains of instability for both countercurrent flow $\left(\Lambda_{\mathrm{r}}>0\right)$, and for cocurrent flow $\left(\Lambda_{\mathrm{r}}<0\right)$. For $0.092<\Lambda_{\mathrm{r}}<10.89$, perturbations are unstable for all values of $H$, and outside this regime there is instability only when $H$ is less than a minimum value or greater than a maximum value.

The range of wave numbers that are unstable can be determined by adding the inertial correction (29) to the viscous solution for the growth rate (17), and determining the wave numbers for which the real part of the resulting growth is positive. This calculation indicates that there are unstable perturbations only for:

$$
k<k_{m}\left(\Lambda_{\mathrm{r}}, \mathrm{Re}\right)
$$




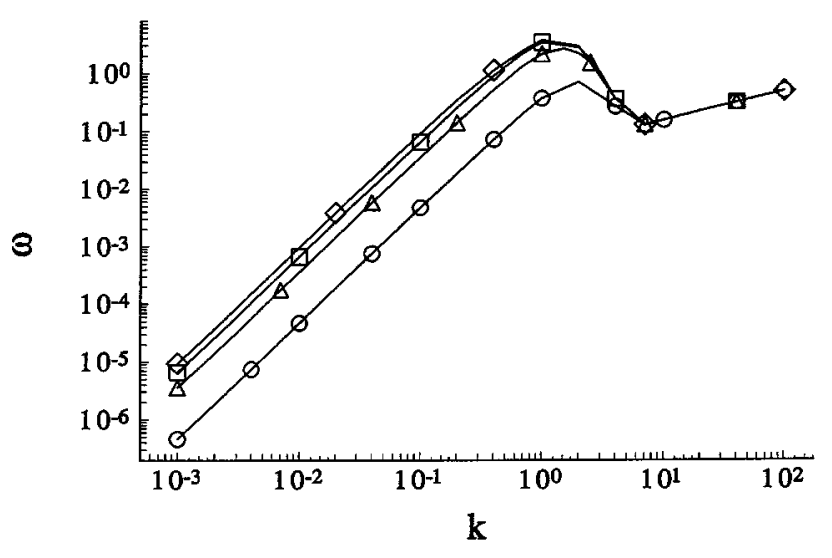

Fig. 6. Variation of the frequency, $\omega$, as a function of wave number, $k$, for $\Sigma=0.1$. (०) $H=0.5 ;(\triangle) H=1.0$; ( $\square)$ $H=1.5 ;(\diamond) H=2.0$.

where

$$
\begin{aligned}
k_{m} & =\left[\frac{\Lambda_{\mathrm{a}} \operatorname{Re}}{10\left(H^{3}+1\right)\left(H^{2}-H+1\right)}\right]^{1 / 2} \\
& \times\left[\left(H^{5}-4 H^{4}+4 H^{3}-2 H^{2}+2 H-2\right)\right. \\
& -6 H \Lambda_{\mathrm{r}}\left(H^{4}-H^{3}+H^{2}-H+1\right) \\
& \left.+H \Lambda_{\mathrm{r}}^{2}\left(1-4 H+4 H^{2}-2 H^{3}+2 H^{4}-2 H^{5}\right)\right]^{1 / 2}
\end{aligned}
$$

while wave numbers with $k>k_{m}$ are stable. The above calculations indicate that fluid inertia has a weakly destabilising effect on long wavelength perturbations in the low Reynolds number limit for certain parameter values of ratio of thickness $H$ and ratio of strain rates $\Lambda_{\mathrm{r}}$. It is of interest to examine the stability of the system when the Reynolds number is raised to finite values. This is determined using numerical methods in the next subsection.

\subsection{Numerical solution}

The domains of instability are determined numerically for the case where $\Lambda_{\mathrm{r}}=0$. The results are expressed as a function of the parameter $\Sigma$ which is defined as $\Sigma \equiv\left(\operatorname{Re} / \Lambda_{\mathrm{a}}\right)=\rho \Gamma R / \eta^{2}$. This ratio is independent of flow parameters and is a function only of fluid and membrane properties.

The numerical method is identical to that used in Srivatsan and Kumaran [6]. There are four constants of integration for the fourth order differential equation (10), out of which two are fixed by the zero velocity conditions at $z=1$ and $z=-H$. Consequently, there are two linearly independent solutions for the velocity fields of fluids $A$ and $B$. The fourth order differential equations for the velocity fields (10) are solved using a fourth order Runge-Kutta integrator. The velocity field in fluid $A$ is determined by starting at the surface $z=1$, and integrating to the interface, while the velocity field in fluid $B$ is obtained by starting at the surface $z=-H$ and integrating to the interface. In addition, it was necessary to use

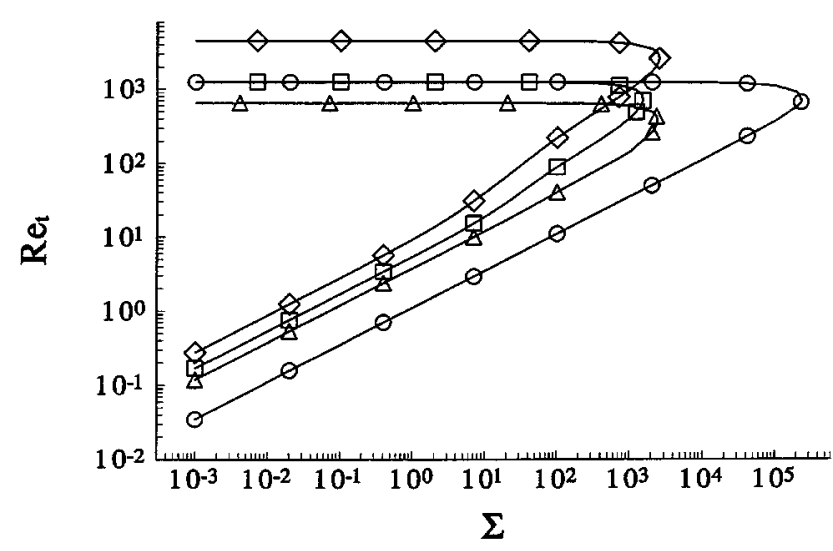

Fig. 7. Variation of the transition Reynolds number, $\mathrm{Re}_{\mathrm{t}}$, as a function $\Sigma$ for $H=1.0$. (॰) $k=0.25$; $(\triangle) k=1.0$; ( $\square) k=2.0$; $(\diamond) k=4.0$.

orthonormalisation techniques due to the stiff nature of the equations. The values for the fundamental solutions at the membrane surface were inserted into the boundary conditions $(13,14)$ to obtain a $4 \times 4$ characteristic matrix. The characteristic equation is obtained by setting the determinant of this matrix equal to zero. The characteristic equation is non-linear in the growth rate $\alpha$, and so it cannot be obtained analytically. Numerical solutions were obtained using Newton-Raphson iteration, and the solutions for finite Reynolds number were determined using a continuation scheme where the zero Reynolds number solutions were taken as the initial guess.

The numerical calculations show that for a constant value of $\Sigma$, the transition Reynolds number $R_{t}$, the Reynolds number at which the system is neutrally stable, increases monotonically with $k$.

$$
\operatorname{Re}_{\mathrm{t}}=k \sqrt{10 \Sigma / \Phi}
$$

where

$$
\Phi=\frac{2-2 H+2 H^{2}-4 H^{3}+4 H^{4}-H^{5}}{\left(H^{3}+1\right)\left(H^{2}-H+1\right)} .
$$

It can easily be verified, by adding up the real parts of the growth rates in the low Reynolds number limit (17) and the low wave number inertial correction (29) that the above transition Reynolds number is in agreement with the asymptotic analyses. Figure 3 shows the variation of $\mathrm{Re}_{\mathrm{t}}$ with $k$ obtained numerically for four values of $\Sigma$. For low values of $k$, the variation of $\log \left(\mathrm{Re}_{\mathrm{t}}\right)$ with $\log (k)$ is linear with slope equal to one, implying that $\operatorname{Re}_{t} \propto k$ for all $\Sigma$ values, and the constant of proportionality is close to the value (30) as predicted by the low wave number analysis. For high values of $k$, the variation of $\log \left(\mathrm{Re}_{\mathrm{t}}\right)$ with $\log (k)$ is linear, but the slope is 1.5 , indicating that $\mathrm{Re}_{\mathrm{t}} \propto k^{1.5}$. The variation of frequency $\omega$ of the neutrally stable mode as a function of $k$ for different values of $\Sigma$ is shown in Figure 4. This graph indicates that $\omega \propto k$ for low values of $k$ and $\omega \propto \sqrt{k}$ for high values of $k$. The dependence of $\mathrm{Re}_{\mathrm{t}}$ on $k$ for different values of $H$ is shown in 


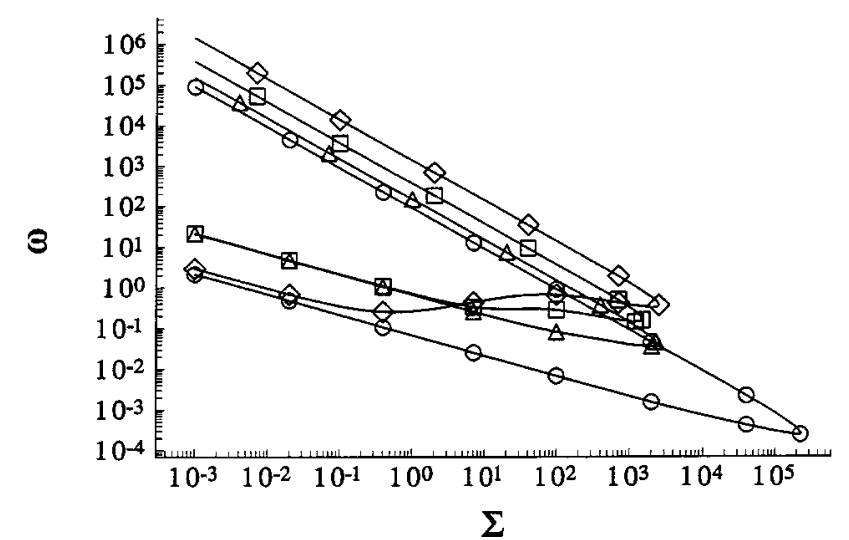

Fig. 8. Variation of the frequency, $\omega$, as a function $\Sigma$ for $H=1.0$. (॰) $k=0.25 ;(\triangle) k=1.0 ;(\square) k=2.0 ;(\diamond) k=4.0$.

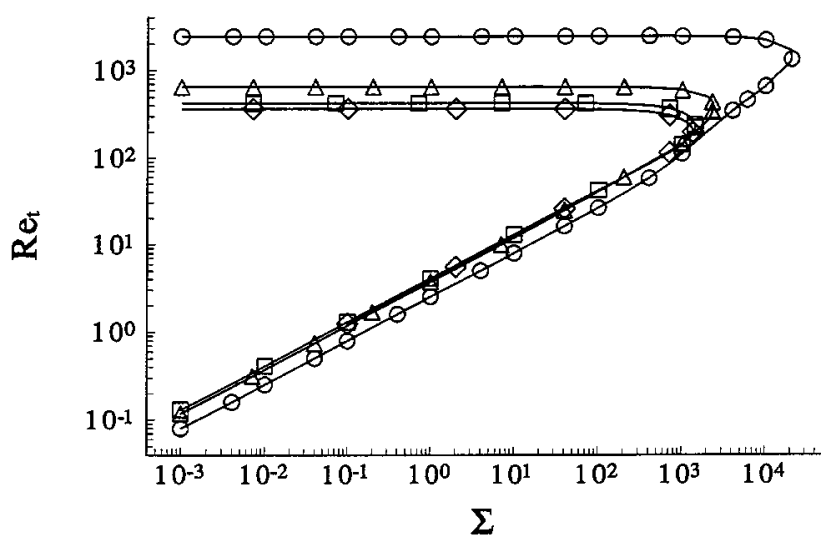

Fig. 9. Variation of the transition Reynolds number, $\mathrm{Re}_{\mathrm{t}}$, as a function $\Sigma$ for $k=1.0$. (०) $H=0.5 ;(\triangle) H=1.0$; ( $\square$ ) $H=1.5 ;(\diamond) H=2.0$.

Figure 5. This scaling relationship of $\mathrm{Re}_{\mathrm{t}}$ with $k$ is identical to that in Figure 3. In addition, it is observed that $\mathrm{Re}_{\mathrm{t}}$ becomes independent of $H$ for $k>4.0$. The frequency of the neutrally stable mode, $\omega$, is shown as a function of $k$ for different values of $H$ at $\Sigma=0.1$ in Figure 6 . The trends observed in this figure are similar to those in Figure 4 for frequency as a function of $k$ at different values of $\Sigma$ for $H=1.0$.

Figure 7 shows the variation of $\operatorname{Re}_{\mathrm{t}}$ with $\Sigma$ for four different values of $k$. The transition Reynolds number first increases as the value of $\Sigma$ is increased, and then undergoes a turning point. Consequently, there is a finite region in the $\operatorname{Re}_{\mathrm{t}}-\Sigma$ space within which the system is unstable, and perturbations are always stable when $\Sigma$ is increased beyond a maximum value. The variation of the frequency $\omega$ of the neutrally stable modes as a function of $\Sigma$ at $H=1.0$ is shown in Figure 8. Figure 9 shows the variation of $\operatorname{Re}_{\mathrm{t}}$ with $\Sigma$ for various values of $H$ at a fixed $k$, and Figure 10 shows the frequency of the neutrally stable modes as a function of $\Sigma$ for different values of $H$ at $k=1.0$. As expected, the qualitative features of these curves are similar to those of Figures 7 and 8 .

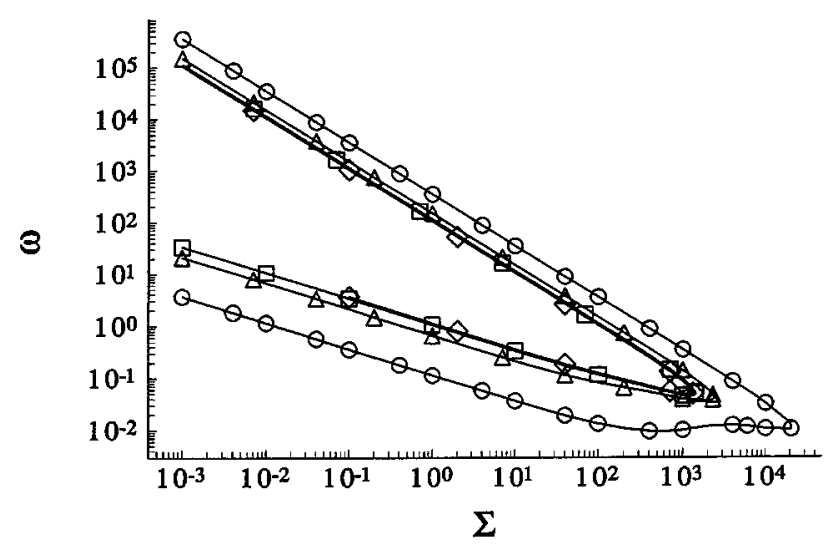

Fig. 10. Variation of the frequency, $\omega$, as a function $\Sigma$ for $k=1.0$. (॰) $H=0.5 ;(\triangle) H=1.0 ;(\square) H=1.5 ;(\diamond) H=2.0$.

\subsection{Surface with no surface tension}

In this subsection, the asymptotic analysis is extended to a surface which has zero surface tension and non-zero curvature elasticity $K$. In this case, the normal stress condition, analogous to $(5)$, becomes

$$
\tau_{z z}^{\mathrm{a}}=\tau_{z z}^{\mathrm{b}}+K \partial_{x}^{4} u .
$$

It is appropriate to scale the velocity by $\left(K / R^{2} \eta\right)$, and the time by $\left(R^{3} \eta / K\right)$. The Reynolds number $\operatorname{Re}$ is defined as before, and the parameters $\Lambda_{\mathrm{a}}=\left(V_{\mathrm{a}} R^{2} \eta / K\right)$ and $\Lambda_{\mathrm{b}}=\left(-V_{\mathrm{b}} R^{2} \eta / K H\right)$. The mean velocity is then given by equation (3), and the linearised equation for the fluid velocity field is given by (10). The boundary conditions (12) at the surfaces $z=1$ and $z=-H$ and the normal and tangential velocity continuity conditions at the surface $z=0$ remain unchanged, but the normal stress condition is

$$
\tilde{\tau}_{z z}^{\mathrm{a}}=\tilde{\tau}_{z z}^{\mathrm{b}}+k^{4} \tilde{u} .
$$

The analysis is carried out with these modifications and the result for the growth rate in the absence of inertia in the limit $k \rightarrow 0$, analogous to (17), is

$$
\begin{aligned}
\alpha= & \frac{-i H k\left(H^{2} \Lambda_{\mathrm{a}}-\Lambda_{\mathrm{b}}\right)}{2\left(H^{3}+1\right)} \\
& +\frac{i k^{3} H^{3}\left[2\left(\Lambda_{\mathrm{a}}+\Lambda_{\mathrm{b}} H\right)(1-H)+5\left(\Lambda_{\mathrm{a}} H^{2}-\Lambda_{\mathrm{b}}\right)\right]}{30\left(H^{3}+1\right)\left(H^{2}-H+1\right)} \\
& +O\left(k^{5}\right) .
\end{aligned}
$$

In contrast to the growth rate (17) for a membrane with surface tension, there is no stabilising term of $O\left(k^{4}\right)$ in the present case because the normal restoring force due to curvature elasticity is $O\left(k^{2}\right)$ smaller than that due to surface tension. The calculations show that the stabilising effect due to curvature elasticity is $O\left(k^{5}\right)$ smaller than the leading order frequency.

The low wave number analysis for the present case is carried out in a manner similar to the analysis of 
Section 2.2, and the calculations show that the leading order and the $O(k)$ correction to the growth rate are identical to (29) and (29), because the surface tension and curvature elasticity do not contribute to the inertial correction to the growth rate at this level of approximation. The destabilising effect due to fluid inertia is the same as that for a surface with surface tension, but the stabilising effect is much smaller. Consequently, the domains of stability shown in Figure 2 are the same for the case where stabilisation is due to curvature elasticity, with the modified definition of the parameters $\Lambda_{\mathrm{a}}$ and $\Lambda_{\mathrm{b}}$. However, the transition Reynolds number is proportional to $k^{2} \sqrt{\Sigma}$, because the destabilising effect is $O\left(\operatorname{Re}^{2} \Sigma k\right)$ smaller than the leading order frequency while the stabilising effect due to curvature elasticity is $O\left(k^{5}\right)$ smaller than the leading order frequency. This dependence is different from (32) for a membrane with surface tension.

\section{Conclusions}

The effect of fluid flow in the stability of the surface fluctuations of a solid membrane separating two Newtonian fluids was analysed using a linear stability analysis. First, the growth rate of fluctuations was calculated analytically in the absence of fluid inertia, and it was found that the real part of the growth rate is always negative, indicating that the fluctuations are always stable at all wave numbers. The fluctuations have the slowest decay rate in the limit $k \rightarrow 0$, where the decay rate is $O\left(k^{3}\right)$ smaller than the leading order frequency. However, in this limit, the inertial terms cause a correction to the growth rate that is $O(k)$ smaller than the leading order frequency. The effect of this correction was calculated using a low wave number $(k \ll 1)$ asymptotic analysis. This indicated that fluctuations could be unstable due to inertial effects for certain values of $\Lambda_{\mathrm{r}}=\left(\Lambda_{\mathrm{b}} / \Lambda_{\mathrm{a}}\right)$ and ratio of thickness $H$.
The asymptotic analysis was extended to finite Reynolds number using numerical analysis for the case where the strain rate in one of the fluid is zero. In the calculations, the Reynolds number for the transition from stable to unstable modes for the fluid with non-zero strain rate, $\mathrm{Re}_{\mathrm{t}}$, was expressed as a function of the parameter $\Sigma=\left(\rho \Gamma R / \eta^{2}\right)$, which is only a function of the fluid and wall parameters and is independent of the fluid velocity. The results of these calculations indicate that the transition Reynolds number first increases as $\Sigma$ is increased, and then undergoes a turning point of infinite slope and any further increase in the transition Reynolds number results in a decrease in $\Sigma$ for the neutrally stable modes. Consequently, perturbations could become unstable only in a finite domain in the $\Sigma$-Re plane, and perturbations are always stable outside this domain.

The authors would like to thank the Department of Science and Technology, Government of India for financial support.

\section{References}

1. M. Sano, M. Kawaguchi, Y.-L. Chen, R.J. Skarlupka, T. Chang, G. Zografi, H. Yu, Rev. Sci. Instrum. 57, 1158 (1986).

2. J.L. Harden, H. Pleiner, P.A. Pincus, J. Chem. Phys. 94, 5208 (1991).

3. V. Kumaran, J. Chem. Phys. 98, 3429 (1993).

4. J.L. Harden, P. Pleiner, Phys. Rev. E 49, 1411 (1994).

5. V. Kumaran, G.H. Fredrickson, P. Pincus, J. Phys. II France 4, 893 (1994).

6. L. Srivatsan, V. Kumaran, J. Phys. II France 7, 947 (1997).

7. V. Kumaran, J. Fluid Mech. 320, 1 (1996); V. Kumaran, J. Fluid Mech. 302, 117 (1995); V. Kumaran, J. Fluid Mech. 293, 259 (1995).

8. P. Krindel, A. Silberberg, J. Coll. Int. Sci. 9, 419 (1987). 